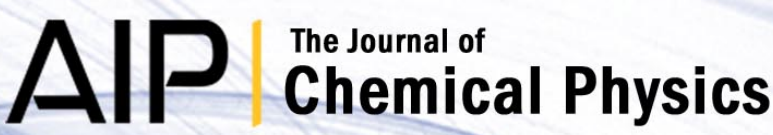

A theoretical study of the conversion of gas phase methanediol to formaldehyde

David R. Kent, Susanna L. Widicus, Geoffrey A. Blake, and William A. Goddard

Citation: J. Chem. Phys. 119, 5117 (2003); doi: 10.1063/1.1596392

View online: http://dx.doi.org/10.1063/1.1596392

View Table of Contents: http://jcp.aip.org/resource/1/JCPSA6/v119/i10

Published by the American Institute of Physics.

Additional information on J. Chem. Phys.

Journal Homepage: http://jcp.aip.org/

Journal Information: http://jcp.aip.org/about/about_the_journal

Top downloads: http://jcp.aip.org/features/most_downloaded

Information for Authors: http://jcp.aip.org/authors

\section{ADVERTISEMENT}

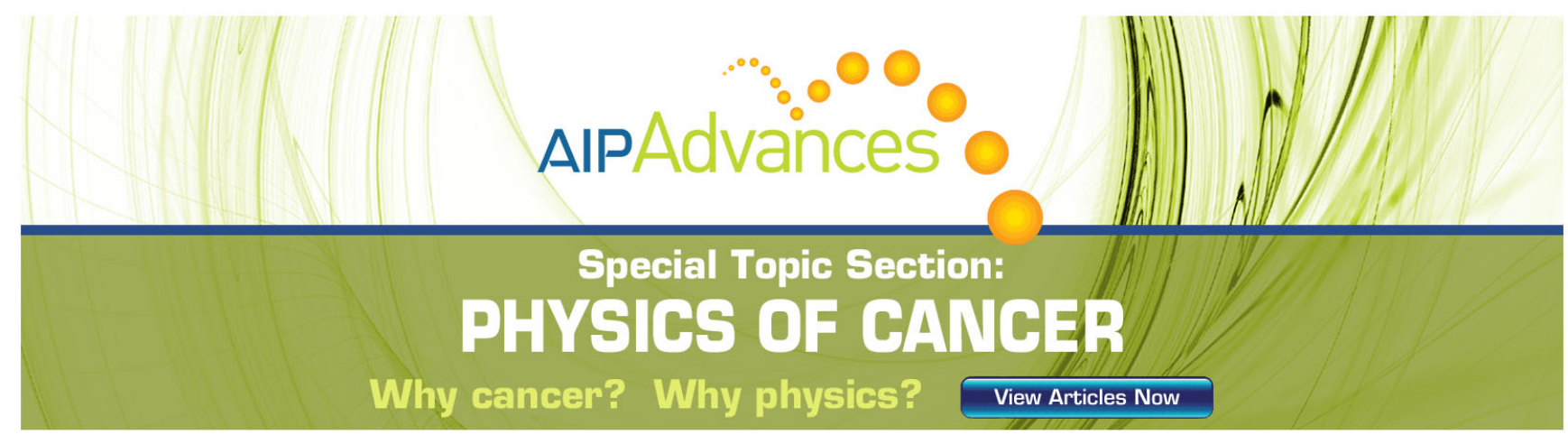




\title{
A theoretical study of the conversion of gas phase methanediol to formaldehyde
}

\author{
David R. Kent IV \\ Materials and Process Simulation Center, Beckman Institute (139-74), Division of Chemistry \\ and Chemical Engineering, California Institute of Technology, Pasadena, California 91125 \\ Susanna L. Widicus \\ Division of Chemistry and Chemical Engineering, California Institute of Technology, \\ Pasadena, California 91125 \\ Geoffrey A. Blake ${ }^{\text {a) }}$ \\ Division of Chemistry and Chemical Engineering, California Institute of Technology, \\ Pasadena, California 91125 and Division of Geological and Planetary Sciences, \\ California Institute of Technology, Pasadena, California 91125 \\ William A. Goddard III \\ Materials and Process Simulation Center, Beckman Institute (139-74), Division of Chemistry and Chemical \\ Engineering, California Institute of Technology, Pasadena, California 91125
}

(Received 28 February 2003; accepted 9 June 2003)

\begin{abstract}
Methanediol, or methylene glycol, is a product of the liquid phase reaction of water and formaldehyde and is a predicted interstellar grain surface species. Detection of this molecule in a hot core environment would advance the understanding of complex organic chemistry in the interstellar medium, but its laboratory spectroscopic characterization is a prerequisite for such observational searches. This theoretical study investigates the unimolecular decomposition of methanediol, specifically the thermodynamic and kinetic stability of the molecule under typical laboratory and interstellar conditions. Methanediol was found to be thermodynamically stable at temperatures of $<100 \mathrm{~K}$, which is the characteristic temperature range for interstellar grain mantles. The infinite-pressure RRKM unimolecular decomposition rate was found to be $<10^{-18} \mathrm{~s}^{-1}$ at $300 \mathrm{~K}$, indicating gas phase kinetic stability for typical laboratory and hot core temperatures. Therefore, both laboratory studies of and observational searches for this molecule should be feasible. (C) 2003 American Institute of Physics. [DOI: 10.1063/1.1596392]
\end{abstract}

\section{INTRODUCTION}

Methanediol, or methylene glycol, has long been known to be the product of the liquid phase reaction of water and formaldehyde. ${ }^{1}$ Analytical studies show that a $5 \%$ by weight aqueous solution of formaldehyde is over $80 \%$ methanediol. ${ }^{1,2}$ The formaldehyde partial pressure above such a solution is 22 Torr, ${ }^{1,3}$ and it is estimated that over $3 \%$ of this vapor is in the form of methanediol. ${ }^{1,2}$ No experimental work has been done, however, to verify that methanediol indeed exists stably in the gas phase.

Small organic monomers such as methanediol are of considerable interest to the field of cosmochemistry. Many oxygen-bearing species, including alcohols, ethers, carboxylic acids, and aldehydes, have been discovered in the interstellar medium, ${ }^{4}$ and many similar species are predicted to exist in detectable amounts. ${ }^{5}$ Methanediol is one of these species, suspected to form in grain surface reactions triggered by the UV or cosmic ray processing of ice

\footnotetext{
a) Author to whom correspondence should be addressed. Electronic mail: gab@gps.caltech.edu
}

mantles. ${ }^{5,6}$ These mantles contain large amounts of water, and formaldehyde has been shown to be a product of such processes, having been detected in cold molecular clouds, protostellar ices, hot core gases, and cometary comae. ${ }^{5}$ It is quite likely, then, that methanediol could form in such an environment.

Observational searches for methanediol should prove useful in the further understanding the chemistry of grain surfaces. Such searches should be straightforward due to the high gas phase abundance predicted for this species in hot core regions, where the temperature of a newly formed star heats the grains sufficiently to evaporate mantle species. ${ }^{7}$ The primary route for destruction of methanediol in hot core environments is unimolecular decomposition, as the low pressure in these regions significantly decreases the possibility for reaction with other species. It is expected to be present in amounts similar to ethanol, or column densities on the order of $10^{15} \mathrm{~cm}^{-2}$. However, such observational searches rely upon gas phase laboratory spectroscopic characterization to provide accurate rest frequencies. Molecular beam experiments can be used to similarly eliminate the possibility of 


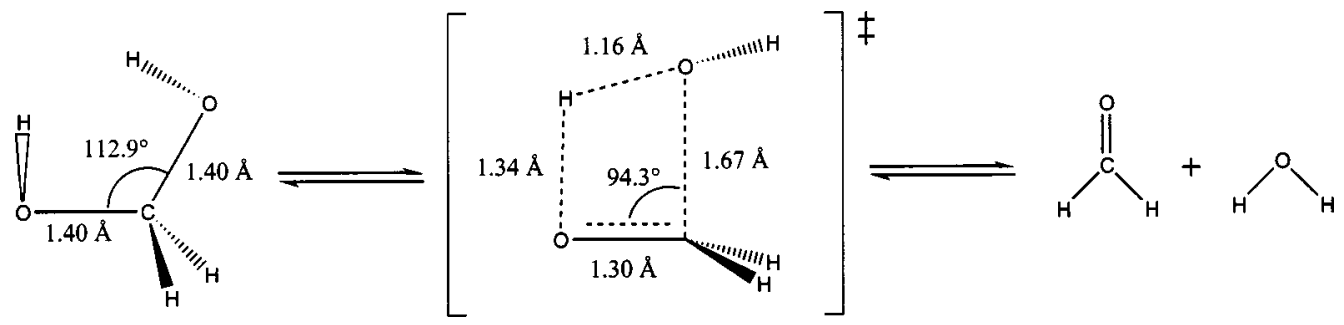

FIG. 1. Unimolecular decomposition of methanediol. Geometries are from MP2/cc-pVTZ calculations.

its reaction with other species in the laboratory. The gas phase thermodynamic and kinetic stability of this species must therefore be verified as the first step in its laboratory investigation.

This work presents a theoretical study that examines the gas phase stability of methanediol, specifically the unimolecular decomposition of this molecule to monomeric formaldehyde and water (Fig. 1).

\section{COMPUTATIONAL DETAILS}

All quantum mechanical calculations presented were performed using MOLPRO version 2000.1. ${ }^{8-10}$ Calculations were done using $\mathrm{HF}$, MP2 $,{ }^{11-13} \mathrm{MP} 4,{ }^{11-13} \mathrm{CCSD},{ }^{11-14}$ $\operatorname{CCSD}(\mathrm{T}),{ }^{11-14}$ and $\mathrm{QCI}(\mathrm{T}){ }^{14}$ with the Dunning double and triple zeta basis sets, cc-pVDZ ${ }^{15}$ and cc-pVTZ. ${ }^{15}$

MP2, MP4, CCSD, CCSD(T), and QCI(T) calculations used optimized MP2 geometries while HF calculations used optimized HF geometries. ${ }^{16-19}$ Geometries for cc-pVDZ single point calculations were obtained using the cc-pVDZ basis; likewise for cc-pVTZ. These geometries can be found in the supplemental material for this paper.

A program, written in the python language ${ }^{20}$ specifically for the purposes of this study, was used for all thermodynamic and kinetic calculations. To simulate finite pressures in the thermodynamic calculations, the ideal gas approximation is used.

TABLE I. Thermodynamics quantities at standard temperature $(298.15 \mathrm{~K})$ and pressure $(1 \mathrm{~atm})$ for the unimolecular decomposition of methanediol to water and formaldehyde (Fig. 1).

\begin{tabular}{lccrcc}
\hline \hline \multicolumn{1}{c}{ Method } & $\Delta E_{\mathrm{el}}(\mathrm{kJ})$ & $\Delta E_{\mathrm{el}+\mathrm{ZPE}}(\mathrm{kJ})$ & $\Delta G(\mathrm{~kJ})$ & $\Delta H(\mathrm{~kJ})$ & $\Delta S(\mathrm{~J})$ \\
\hline HF/cc-pVDZ & 55.3 & 28.7 & -9.2 & 36.5 & 153.4 \\
MP2/cc-pVDZ & 56.3 & 30.1 & -8.1 & 38.0 & 154.5 \\
MP4/cc-pVDZ & 50.6 & 24.6 & -13.5 & 32.3 & 153.6 \\
CCSD/cc-pVDZ & 54.7 & 28.6 & -9.5 & 36.4 & 154.1 \\
CCSD(T)/cc-pVDZ & 52.6 & 27.0 & -11.0 & 34.7 & 153.1 \\
QCI(T)/cc-pVDZ & 52.2 & 26.6 & -11.4 & 34.2 & 153.2 \\
HF/cc-pVTZ & 50.4 & 23.8 & -13.9 & 31.5 & 152.6 \\
MP2/cc-pVTZ & 61.7 & 33.6 & -4.2 & 41.2 & 152.3 \\
MP4/cc-pVTZ & 56.8 & 31.6 & -6.2 & 39.0 & 151.4 \\
CCSD/cc-pVTZ & 60.6 & 34.7 & -3.3 & 42.4 & 153.4 \\
CCSD(T)/cc-pVTZ & 59.4 & 33.8 & -4.2 & 41.5 & 153.1 \\
QCI(T)/cc-pVTZ & 59.1 & 33.5 & -4.5 & 41.1 & 153.2 \\
\hline
\end{tabular}

${ }^{a}$ Vibrational frequencies from cc-pVDZ.

\section{RESULTS AND DISCUSSION}

Calculations of the thermodynamic quantities for the unimolecular decomposition of methanediol into formaldehyde and water (Table I) show that the decomposition is entropically driven with a free energy change of between -3 and $-7 \mathrm{~kJ}$ at standard temperature $(298.15 \mathrm{~K})$ and pressure (1 atm). A diagram showing the relative energies of methanediol, the transition state, and the products is shown in Fig. 2.

At a temperature between 100 and $300 \mathrm{~K}$ (Table II), depending on pressure, the decomposition switches from being spontaneous to nonspontaneous with lower temperatures being nonspontaneous. Therefore, at low temperatures $(<100 \mathrm{~K})$ methanediol is thermodynamically stable while at higher temperatures $(>300 \mathrm{~K})$ methanediol decomposes into formaldehyde and water.

An estimate of an upper bound on the unimolecular decomposition rate can be calculated using either RRKM theory at infinite pressure or transition state theory (TST). Both theories give the same expression for the rate, $k_{\text {uni }}^{\infty}$,

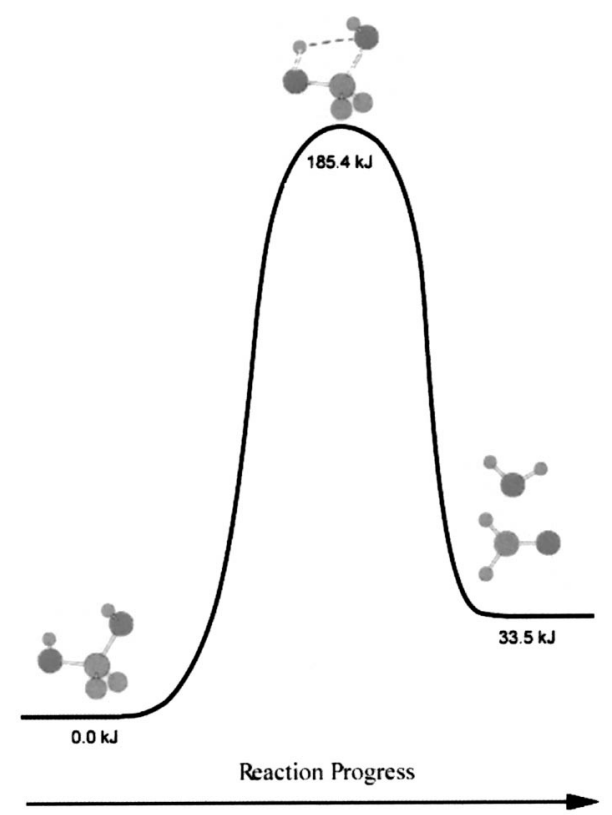

FIG. 2. Relative enthalpies at $0 \mathrm{~K}\left(\Delta E_{\mathrm{el}+\mathrm{ZPE}}\right)$ for the decomposition of methanediol. The electronic energies and vibrational frequencies were evaluated using QCI(T)/cc-pVTZ and QCI(T)/cc-pVDZ, respectively. 
TABLE II. Temperatures in Kelvin for which $\Delta G=0$ for the unimolecular decomposition of methanediol to water and formaldehyde (Fig. 1).

\begin{tabular}{lcccc}
\hline \hline \multicolumn{1}{c}{ Method } & $10^{-6}$ atm & $10^{-4}$ atm & $10^{-2}$ atm & $10^{0}$ atm \\
\hline HF/cc-pVDZ & 132 & 155 & 188 & 237 \\
MP2/cc-pVDZ & 137 & 161 & 195 & 245 \\
MP4/cc-pVDZ & 115 & 136 & 165 & 209 \\
CCSD/cc-pVDZ & 131 & 155 & 187 & 236 \\
CCSD(T)/cc-pVDZ & 125 & 147 & 179 & 225 \\
QCI(T)/cc-pVDZ & 123 & 145 & 176 & 222 \\
HF/cc-pVTZ & 112 & 133 & 162 & 205 \\
MP2/cc-pVTZ & 151 & 178 & 215 & 271 \\
MP4/cc-pVTZ & 143 & 169 & 204 & 257 \\
CCSD/cc-pVTZ & 155 & 183 & 220 & 276 \\
\hline \hline
\end{tabular}

$$
k_{\mathrm{uni}}^{\infty}=\frac{k_{B} T}{h} \frac{q_{r}^{\ddagger} q_{v}^{\ddagger}}{q_{r} q_{v}} e^{-E_{a} / k_{B} T},
$$

where $k_{B}$ is the Boltzmann constant, $h$ is Planck's constant, $T$ is the temperature, $E_{a}$ is the activation energy, and $q_{r}, q_{v}, q_{r}^{\ddagger}$, and $q_{v}^{\ddagger}$ are the rotational and vibrational partition functions for methanediol and the transition state. Table III shows the calculated rates for 300, 500, and $1000 \mathrm{~K}$. Tunneling of the hydroxyl proton is not expected due to the anticipated strength of the hydrogen bond and has therefore not been included in the RRKM calculations.

Excluding the HF and cc-pVDZ calculations, which are of lower quality than the other approaches, an upper bound on the unimolecular decomposition rate of roughly $10^{-22}-10^{-18} \mathrm{~s}^{-1}$ at $300 \mathrm{~K}$ can be established. Similar bounds of $10^{-8}-10^{-6} \mathrm{~s}^{-1}$ at $500 \mathrm{~K}$ and $10^{3}-10^{4} \mathrm{~s}^{-1}$ at $1000 \mathrm{~K}$ can be calculated. These bounds correspond to a lifetime lower limit of roughly $10^{10}-10^{14} \mathrm{yr}$ at $300 \mathrm{~K}$, $10^{-2}-10^{0} \mathrm{yr}$ at $500 \mathrm{~K}$, and $10^{-4}-10^{-3} \mathrm{~s}$ at $1000 \mathrm{~K}$, indicating that methanediol is kinetically stable at temperatures $\$ 300 \mathrm{~K}$ (Table III).

The results of this study indicate that the characterization of methanediol under typical laboratory conditions should be

TABLE III. Activation energy and infinite-pressure RRKM unimoleculardecomposition rates for methanediol conversion to water and formaldehyde (Fig. 1). These rates are an upper bound on the observed rate.

\begin{tabular}{lcccc}
\hline \hline & & \multicolumn{3}{c}{$k_{\text {uni }}^{\infty}\left(\mathrm{s}^{-1}\right)$} \\
\cline { 3 - 5 } \multicolumn{1}{c}{ Method } & $E_{a}(\mathrm{~kJ} / \mathrm{mol})$ & $300 \mathrm{~K}$ & $500 \mathrm{~K}$ & $1000 \mathrm{~K}$ \\
\hline HF/cc-pVDZ & 241.9 & $8.8 \times 10^{-30}$ & $9.1 \times 10^{-13}$ & $6.4 \times 10^{+00}$ \\
MP2/cc-pVDZ & 182.2 & $2.3 \times 10^{-19}$ & $1.7 \times 10^{-06}$ & $9.9 \times 10^{+03}$ \\
MP4/cc-pVDZ & 182.0 & $2.5 \times 10^{-19}$ & $1.8 \times 10^{-06}$ & $1.0 \times 10^{+04}$ \\
CCSD/cc-pVDZ & 199.2 & $2.4 \times 10^{-22}$ & $2.6 \times 10^{-08}$ & $1.1 \times 10^{+03}$ \\
CCSD(T)/cc-pVDZ & 187.6 & $2.5 \times 10^{-20}$ & $4.3 \times 10^{-07}$ & $4.5 \times 10^{+03}$ \\
QCI(T)/cc-pVDZ & 186.3 & $4.3 \times 10^{-20}$ & $6.1 \times 10^{-07}$ & $5.5 \times 10^{+03}$ \\
HF/cc-pVTZ & 240.5 & $1.4 \times 10^{-29}$ & $1.1 \times 10^{-12}$ & $6.4 \times 10^{+00}$ \\
MP2/cc-pVTZ & 179.6 & $5.8 \times 10^{-19}$ & $2.7 \times 10^{-06}$ & $1.1 \times 10^{+04}$ \\
MP4/cc-pVTZ & 179.2 & $7.7 \times 10^{-19}$ & $3.5 \times 10^{-06}$ & $1.4 \times 10^{+04}$ \\
CCSD/cc-pVTZ & 199.6 & $2.0 \times 10^{-22}$ & $2.4 \times 10^{-08}$ & $1.0 \times 10^{+03}$ \\
CCSD(T)/cc-pVTZ & 186.7 & $3.6 \times 10^{-20}$ & $5.3 \times 10^{-07}$ & $5.0 \times 10^{+03}$ \\
QCI(T)/cc-pVTZ & 185.4 & $6.2 \times 10^{-20}$ & $7.6 \times 10^{-07}$ & $6.1 \times 10^{+03}$ \\
\hline
\end{tabular}

${ }^{a}$ Vibrational frequencies from cc-pVDZ. straightforward, as it is both thermodynamically and kinetically stable at typical experimental conditions $(300 \mathrm{~K}$, $\left.10^{-5} \mathrm{~atm}\right)$. In addition, methanediol should also be stable in interstellar hot core environments, where temperatures are typically between 100 and $300 \mathrm{~K}$ and pressures are $<10^{-18}$ atm. (See the Supplemental Tables IV-VII. ${ }^{21}$ ) Indeed, with a lifetime lower limit of $\sim 10^{10} \mathrm{yr}$ compared to the typical hot core lifetime of $\sim 10^{6} \mathrm{yr}$, methanediol should be observable at the abundances predicted by current hot core models. ${ }^{21}$

Laboratory characterization of the methanediol rotational spectrum is underway. Initial millimeter wavelength experiments utilize a direct absorption flow cell apparatus, where the vapor from a $5 \%$ by weight aqueous solution of formaldehyde is being continuously flowed through the cell. Additional experiments will include microwave studies of lower energy rotational transitions with a Fabry-Perot cavity pulsed Fourier transform microwave spectrometer, also known as a Flygare instrument. The results of these studies will then be used to guide observational searches. ${ }^{21}$

\section{ACKNOWLEDGMENTS}

D.R.K. is grateful for a graduate fellowship from The Fannie and John Hertz Foundation. S.L.W. and G.A.B. thank the NASA Exobiology and SARA programs, Grant Nos. NAG5-8822 and NAG5-11423. We gratefully acknowledge the support and services rendered by the CIT staff. The computational resources at the MSC were provided by the NSF (CHE-99MRI), IBM (SUR Grant), ARO-DURIP, and ONRDURIP. Other support for the MSC came from NIH, Chevron Texaco, GM, Seiko-Epson, Beckman Institute, Asahi Kasei, Nippon Steel, and DOE ASCI ASAP.

${ }^{1}$ F. J. Walker, Formaldehyde (Reinhold, New York, 1964).

${ }^{2}$ S. Bezzi, N. Dallaporta, G. Giacommetti, and A. Ilicito, Gazz. Chim. Ital. 81, 915 (1951).

${ }^{3}$ E. L. Piret and M. W. Hall, Ind. Eng. Chem. 40, 661 (1948).

${ }^{4}$ M. Ohishi, "Observations of hot cores," in Molecules in Astrophysics, No. 178 in IAU Symposium, edited by E. F. van Dishoeck (Kluwer, Dordrecht, 1997), p. 61.

${ }^{5}$ P. Erenfreund and S. B. Charnley, Annu. Rev. Astron. Astrophys. 38, 427 (2000).

${ }^{6} \mathrm{~S}$. Charnley, "Interstellar organic chemistry," in The Proceedings of the Workshop The Bridge Between the Big Bang and Biology (Consiglio Nazionale delle Ricerche, Italy, 1997).

${ }^{7}$ E. F. van Dishoeck and G. A. Blake, Annu. Rev. Astron. Astrophys. 36, 317 (1998).

${ }^{8}$ R. D. Amos, A. Bernhardsson, A. Berning et al., MOLPRO, a package of $a b$ initio programs designed by H.-J. Werner and P. J. Knowles, version 2000.1, 2000.

${ }^{9}$ R. Lindh, U. Ryu, and B. Liu, J. Chem. Phys. 95, 5889 (1991).

${ }^{10}$ G. Rauhut, A. El Azhary, F. Eckert, U. Schumann, and H.-J. Werner, Spectrochim. Acta, Part A 55, 651 (1999).

${ }^{11}$ C. Hampel and H.-J. Werner, J. Chem. Phys. 104, 6286 (1996).

${ }^{12}$ G. Hetzer, P. Pulay, and H.-J. Werner, Chem. Phys. Lett. 290, 143 (1998).

${ }^{13}$ M. Schutz, G. Hetzer, and H.-J. Werner, J. Chem. Phys. 111, 5691 (1999).

${ }^{14}$ C. Hampel, K. Peterson, and H.-J. Werner, Chem. Phys. Lett. 190, 1 (1992).

${ }^{15}$ T. H. Dunning, J. Chem. Phys. 90, 1007 (1989).

${ }^{16}$ R. Lindh, Theor. Chim. Acta 85, 423 (1993).

${ }^{17}$ A. El Azhary, G. Rauhut, P. Pulay, and H.-J. Werner, J. Chem. Phys. 108, 5185 (1998)

${ }^{18}$ F. Eckert, P. Pulay, and H.-J. Werner, J. Comput. Chem. 18, 1473 (1997). 
${ }^{19}$ F. Eckert and H.-J. Werner, Theor. Chim. Acta 100, 21 (1998).

${ }^{20}$ http://www.python.org

${ }^{21}$ See EPAPS Document No. E-JCPSA6-119-010333 for Supplemental

Tables IV-VII. A direct link to this document may be found in the online article's HTML reference section. The document may also be reached via the EPAPS homepage (http://www.aip.org/pubservs/epaps.html) or from ftp.aip.org in the directory /epaps/. See the EPAPS homepage for more information. 